\title{
Analytic solutions to a strongly nonlinear Vlasov equation.
}

\author{
P.E. Jabin, A. Nouri \\ University of Nice-Sophia Antipolis, France \\ Aix-Marseille University, France
}

\begin{abstract}
We prove the existence for short times of analytic solutions to a Vlasov type equation. The corresponding model is one-dimensional but uses a quite singular force term which involves a full derivative in $x$ of the macroscopic density, making the existence of solutions a difficult question.
\end{abstract}

Résumé. Nous démontrons l'existence en temps petit de solution analytique à une équation de type Vlasov. Le modèle considéré est mono-dimensionnel mais le terme de force correspondant fait intervenir une dérivée complète de la densité macroscopique. Ceci rend la question de l'existence de solution particulièrement délicate.

\section{Version française abrégée.}

Le but de cette note est de démontrer l'existence de solutions analytiques en temps petit pour l'équation cinétique

$$
\begin{aligned}
& \partial_{t} f+v \partial_{x} f-\partial_{x}\left(\int f(t, x, v) d v\right) \partial_{v} f=0, \quad t \geq 0, x, v \in \mathbb{R} \\
& f(t=0, x, v)=f_{i}(x, v) .
\end{aligned}
$$

Cette équation peut être obtenue à partir de modèles de plasma sans collision, sous certaines asymptotiques. Nous renvoyons à [3] et [6] et aux références que ces deux articles citent pour une présentation plus détaillée de la dérivation de cette équation.

L'équation (1) est de type Vlasov et hamiltonienne. A l'inverse du modèle plus classique de Vlasov-Poisson où l'énergie potentielle dans le hamiltonien présente un gain de deux dérivées par rapport à la densité macroscopique, ici il n'existe aucun gain en dérivée. Même en dimension 1, (1) est donc particulièrement singulière, deux ordres de plus que Vlasov-Poisson et un ordre de plus que Vlasov-Maxwell.

Il est désormais bien connu comment prouver l'existence de solutions fortes ou classiques pour le système de Vlasov-Poisson, même en dimension 3. Parmi de nombreuses contributions importantes, nous renvoyons plus spécifiquement à [1] pour des données initiales petites, à [9], [11], [12] pour des données quelconques en temps grand et à [2] pour le cas analytique. Dans le cas plus singulier de Vlasov-Maxwell, l'existence de solutions classiques est connue en temps

\footnotetext{
${ }^{1}$ E-mail addresses: jabin@math.unice.fr (P.E. Jabin), nouri@cmi.univ-mrs.fr (A.Nouri).

${ }^{2} 2000$ Mathematics Subject Classification. 41A60, 76P05, 82A70, 78A35.

${ }^{3}$ Key words. Plasmas, electroneutrality equation, Cauchy problem.
} 
grand en dimension 1 ou 2 ( $\mathrm{cf}[7]$ ou [8]). En dimension 3, seuls des résultats en temps petit ont été obtenus (voir par exemple [4]) ou pour des solutions faibles, cf [5].

De par sa nature très singulière, le seul résultat d'existence que nous connaissions pour (1) (même pour des temps courts ou des solutions faibles) est dû à [3] où les auteurs ne peuvent traiter qu'un type particulier de données initiales, constantes par morceaux. Cette étude montre clairement que l'équation n'est pas quasi-linéaire contrairement aux modèles cinétiques habituels ; le terme $\partial_{x}\left(\int f(t, x, v) d v\right) \partial_{v} f$ est en effet du même ordre que le terme de transport $v \partial_{x} f$. Ceci rend douteuse la possibilité d'obtenir une théorie d'existence pour des données initiales générales ( $C^{\infty}$ par exemple) sans condition de forme.

Ici nous nous limitons à des données initiales analytiques et nous démontrons

Théorème. Soit $f_{i}$ une fonction analytique dont toutes les dérivées sont bornées en position et décroissent plus que polynomialement en vitesse, alors il existe un temps $T$ et une unique fonction analytique $f$ sur $[0, T] \times \mathbb{R}^{2}$ solution de $(1)$.

Le temps d'existence $T$ dépend de la donnée initiale, et en fait de sa régularité exacte et pas seulement de sa taille. En particulier, si l'on prend $f_{i}=\varepsilon \bar{f}_{i}$ en faisant $\varepsilon \rightarrow 0$, alors notre preuve ne garantit pas que $T \rightarrow \infty$.

Remarquons que les solutions analytiques d'équations cinétiques ne permettent pas d'observer plusieurs phénomènes d'instabilité. De ce fait ce résultat n'est pas très satisfaisant, bien moins que si l'on supposait seulement $f_{i}$ dans $C^{\infty}$ par exemple. En particulier, la preuve ne dépend pas du signe du terme de force et s'appliquerait donc de la même façon à

$$
\partial_{t} f+v \partial_{x} f+\partial_{x}\left(\int f(t, x, v) d v\right) \partial_{v} f=0 .
$$

Mais le terme d'interaction dans cette dernière équation est attractif et celle-ci devrait donc être plus difficile à traiter que (1).

Mentionnons finalement que les outils développés dans [10] permettent d'obtenir le même résultat que celui présenté ici. L'intérêt de notre preuve est d'être beaucoup plus simple.

\section{Introduction.}

The aim of this note is to prove the existence of analytic solution in short time for the kinetic equation

$$
\begin{aligned}
& \partial_{t} f+v \partial_{x} f-\partial_{x}\left(\int f(t, x, v) d v\right) \partial_{v} f=0, \quad t \geq 0, x, v \in \mathbb{R} \\
& f(t=0, x, v)=f_{i}(x, v) .
\end{aligned}
$$

This equation is obtained under certain scalings from collisionless plasma models, we refer to [3] or [6] and the references therein for a more detailed presentation of the equation.

Eq. (2) is Hamiltonian of Vlasov type. Contrary to the more classical Vlasov-Poisson model where the potential energy in the Hamiltonian gains two derivatives with respect to the macroscopic density, here no derivatives at all are gained. Even in dimension one, Eq. (2) is two orders more singular than Vlasov-Poisson and one order more than Vlasov-Maxwell.

Results showing the existence of strong or classical solutions to the Vlasov-Poisson system are now well known, even in dimension 3; among many important contributions, we refer to [1] for small initial data, to [9], [11] and [12] for long time existence with any initial data, and to [2] 
for existence of analytic solutions. For Vlasov-Maxwell, existence of classical solutions is known in dimension 1 or 2 for long time, see [7] or [8]. In dimension 3, only short time results are for the moment available, see for instance [4]; results in large time concern only weak solutions, see $[5]$.

Due to its highly singular nature, the only existence result (even for short times or weak solutions) we know of for (2) is obtained in [3] where the authors only consider a particular form of initial data : the so-called water bags where the initial data is piecewise constant. From this study, it is clear that the equation is not quasi-linear anymore, the term $\partial_{x}\left(\int f(t, x, v) d v\right) \partial_{v} f$ being of the same order as the usual transport $v \partial_{x} f$. This casts some doubts whether for general initial data (for instance $C^{\infty}$ ) without a shape assumption, a theory of well posedness could even be hoped for.

Here we consider only analytical initial data and show (see below for a precised result)

Theorem 1. Let $f_{i}$ be an analytic function with all derivatives bounded in position and exponential decay at infinity in velocity. Then there exist a time $T$ and a unique analytic function $f$ in $[0, T] \times \mathbb{R}^{2}$ which solves Eq. (2).

Note that the time of existence $T$ depends on the initial data itself, actually on how regular it is and not just on how large it is (again see below for a precise result). In particular, if one considers $f_{i}=\varepsilon \bar{f}_{i}$ and lets $\varepsilon \rightarrow 0$, then our proof does not guarantee that the time of existence $T \rightarrow \infty$.

Analytic solutions to kinetic equations notoriously miss many instability phenomena of those equations. Because of that this result is still not very satisfactory (much less than well posedness in $C^{\infty}$ for instance). In particular, it does not depend on the sign of the force term and would apply equally to

$$
\partial_{t} f+v \partial_{x} f+\partial_{x}\left(\int f(t, x, v) d v\right) \partial_{v} f=0 .
$$

The interaction in this last equation is attractive and should be much more difficult to handle than for (2). However at the level of analytic solutions, the difference is not seen...

Finally we mention that the tools put in place in [10] imply the same result as the one in this note. The interest of our proof is that it is quite simple and does not require the heavy machinery put in place in [10].

\section{The result}

For any function $g(t, x, v)$ and positive real numbers $\left(\lambda_{0}, K\right)$, denote by

$$
\begin{array}{r}
|g|_{\lambda}:=\sum_{(k, l) \in \mathbb{N}^{2}} \frac{|\lambda|^{k+l}}{k ! l !}\left\|\partial_{x}^{k} \partial_{v}^{l} g\right\|_{\infty}, \quad|g|_{\lambda, a}:=\frac{\partial^{a}}{\partial \lambda^{a}}|g|_{\lambda}, \quad a \in \mathbb{N}, \\
\lambda(t):=\lambda_{0}-(1+K) t, \quad H_{g}(t):=\sum_{a \in \mathbb{N}} \frac{|g|_{\lambda(t), a}(t)}{(a !)^{2}}, \quad \tilde{H}_{g}(t):=\sum_{a \in \mathbb{N}} a^{2} \frac{|g|_{\lambda(t), a}(t)}{(a !)^{2}} .
\end{array}
$$

The main result of this note is given by the following theorem

Theorem 2. Let $\alpha$ be a positive function such that

$$
\int \alpha(v) d v \leq 1, \quad \sum_{k \in \mathbb{N}} \frac{1}{(k !)^{2}} \sum_{l \geq k} \frac{\lambda_{0}^{l-k}}{(l-k) !}\left|\left(\frac{\alpha^{\prime}}{\alpha}\right)^{(l)}\right|_{\infty} \leq \tilde{\gamma}
$$


for some finite $\tilde{\gamma}$ and $\lambda_{0}$. Assume that $h_{0}:=\sum \frac{1}{(a !)^{2}}\left|\frac{\rho_{i}}{\alpha}\right|_{\lambda_{0}, a}$ is finite, where $\rho_{i}(x)=\int f_{i}(x, v) d v$. Let $M>0$ and $\tilde{M}:=\frac{1}{24+\tilde{\gamma}} \log \frac{M}{h_{0}}$ be such that

$$
\tilde{M}+\tilde{\gamma} M<1
$$

Let

$$
T \in] 0, \min \left(\frac{|\log (\tilde{M}+\tilde{\gamma} M)|}{\tilde{\gamma} M}, \lambda_{0}\left(1+\max \left(2 \lambda_{0}+8 M, \frac{2 M}{\tilde{M}}, \tilde{\gamma} \tilde{M}+5 M+\tilde{\gamma} M+1\right)\right)^{-1}\right)[.
$$

There is a unique analytic solution $f$ defined on $[0, T]$, such that $\sup _{t \in[0, T]} H_{\rho}(t) \leq M$ and $\int_{0}^{T} \tilde{H}_{\rho}(t) d t \leq \tilde{M}$ to Eq. (2).

Note that $\alpha(v)=\frac{1}{1+v^{2}}$ satisfies the assumptions for some $\lambda_{0}$ small enough, as $\alpha^{\prime} / \alpha=2 v /(1+$ $v^{2}$ ) and hence

$$
\left|\left(\frac{\alpha^{\prime}}{\alpha}\right)\right|_{\infty} \leq C^{l} l !
$$

Therefore Th. 2 indeed implies Th. 1. It is more precise though and actually allows for a much weaker decay in velocity.

\section{Proof of Theorem 2}

Consider the space of functions bounded in $t$ and analytic in $x$, with the norm

$$
\|\sigma\|=\sup _{t \in[0, T]} H_{\sigma}(t)+\int_{0}^{T} \tilde{H}_{\sigma}(t) d t
$$

Let $\Phi$ be the map mapping $\sigma \in \tilde{K}:=\left\{\sigma ; \sup _{t \in[0, T]} H_{\sigma}(t) \leq M, \quad \int_{0}^{T} \tilde{H}_{\sigma}(t) d t \leq \tilde{M}\right\}$ into $\rho$ defined by $\rho(t, x)=\int \alpha(v) g(t, x, v) d v$, where $g$ is the solution of

$$
\partial_{t} g+v \cdot \partial_{x} g-\partial_{x} \sigma \cdot\left(\partial_{v} g+\gamma g\right)=0
$$

with initial condition $g_{i}:=\frac{f_{i}}{\alpha}$ and $\gamma:=\frac{\alpha^{\prime}}{\alpha}$. In the first step of the proof, it is shown that $\Phi$ maps $\tilde{K}$ into $\tilde{K}$. In the second step, it is shown that $\Phi$ is a contraction for the $\|\cdot\|$ norm.

First step.

Lemma 3. Let $\sigma(t, x)$ with $\sum_{a \in \mathbb{N}} \frac{1}{(a !)^{2}}|\sigma|_{\lambda, a}$ finite be given. For any solution $g$ to (5), it holds that

$$
\partial_{t}|g|_{\lambda, a} \leq a|g|_{\lambda, a}+\lambda|g|_{\lambda, a+1}+\frac{\partial^{a}}{\partial \lambda^{a}}\left(|\sigma|_{\lambda, 1}|g|_{\lambda, 1}+|\sigma|_{\lambda, 1}|\gamma|_{\lambda}|g|_{\lambda}\right)
$$

Proof of Lemma 3 Differentiate $(5) k$ (resp. $l$ ) times with respect to $x$ (resp. $v$ ). Then,

$$
\begin{array}{r}
\partial_{t}\left(\partial_{x}^{k} \partial_{v}^{l} g\right)+v \cdot \partial_{x}\left(\partial_{x}^{k} \partial_{v}^{l} g\right)-\partial_{x} \sigma \cdot \partial_{v}\left(\partial_{x}^{k} \partial_{v}^{l} g\right)=-l \partial_{x}^{k+1} \partial_{v}^{l-1} g \\
+\sum_{m=0}^{k-1} C_{k}^{m}\left(\partial_{x}^{k-m+1} \sigma\right)\left(\partial_{x}^{m} \partial_{v}^{l+1} g\right)+\sum_{m=0}^{k} C_{k}^{m}\left(\partial_{x}^{k-m+1} \sigma\right)\left(\partial_{x}^{m} \partial_{v}^{l}(\gamma g)\right) .
\end{array}
$$


By convention the terms where a negative index appears are assumed to vanish. Hence,

$$
\begin{array}{r}
\frac{d}{d t}|g|_{\lambda, a} \leq \sum_{k+l \geq a} \frac{|\lambda|^{k+l-a}}{k !(l-1) !} \frac{(k+l) !}{(k+l-a) !}\left\|\partial_{x}^{k+1} \partial_{v}^{l-1} g\right\|_{\infty} \\
+\sum_{k+l \geq a} \frac{\partial^{a}}{\partial \lambda^{a}}\left(\lambda^{k+l}\right) \frac{1}{l !} \sum_{m \leq k-1} \frac{1}{m !(k-m) !}\left\|\partial_{x}^{m} \partial_{v}^{l+1} g\right\|_{\infty}\left\|\partial_{x}^{k-m+1} \sigma\right\|_{\infty} \\
+\sum_{k+l \geq a} \frac{\partial^{a}}{\partial \lambda^{a}}\left(\lambda^{k+l}\right) \frac{1}{l !} \sum_{m \leq k} \frac{1}{m !(k-m) !}\left\|\partial_{x}^{m} \partial_{v}^{l}(\gamma g)\right\|_{\infty}\left\|\partial_{x}^{k-m+1} \sigma\right\|_{\infty} .
\end{array}
$$

The first term in the right hand side of (6) is equal to

$$
\begin{array}{r}
\sum_{k^{\prime}+l^{\prime} \geq a} \frac{|\lambda|^{k^{\prime}+l^{\prime}-a}}{\left(k^{\prime}-1\right) ! l^{\prime} !} \frac{\left(k^{\prime}+l^{\prime}\right) !}{\left(k^{\prime}+l^{\prime}-a\right) !}\left\|\partial_{x}^{k^{\prime}} \partial_{v}^{l^{\prime}} g\right\|_{\infty}=\sum_{k+l \geq a} k \frac{|\lambda|^{k+l-a}}{k ! l !} \frac{(k+l) !}{(k+l-a) !}\left\|\partial_{x}^{k} \partial_{v}^{l} g\right\|_{\infty} \\
\leq a \sum_{k+l \geq a} \frac{|\lambda|^{k+l-a}}{k ! l !} \frac{(k+l) !}{(k+l-a) !}\left\|\partial_{x}^{k} \partial_{v}^{l} g\right\|_{\infty}+\sum_{k+l \geq a+1} \frac{|\lambda|^{k+l-a}}{k ! l !} \frac{(k+l) !}{(k+l-a-1) !}\left\|\partial_{x}^{k} \partial_{v}^{l} g\right\|_{\infty} \\
=a|g|_{\lambda, a}+\lambda|g|_{\lambda, a+1} .
\end{array}
$$

The second term in the right hand side of (6) is equal to $\frac{\partial^{a}}{\partial \lambda^{a}} Y$, where

$$
Y \leq|\sigma|_{\lambda, 1}\left(|g|_{\lambda, 1}-\sum_{m \geq 1} \frac{\lambda^{m-1}}{(m-1) !}\left\|\partial_{x}^{m} g\right\|_{\infty}-\sum_{m \geq 1, l \geq 0} \frac{\lambda^{m+l}}{(m-1) !(l+1) !}\left\|\partial_{x}^{m} \partial_{v}^{l+1} g\right\|_{\infty}\right) .
$$

Similarly, the third term in the right hand side of (6) is equal to $\frac{\partial^{a}}{\partial \lambda^{a}} \tilde{Y}$, where

$$
\frac{\partial^{a}}{\partial \lambda^{a}} \tilde{Y} \leq \frac{\partial^{a}}{\partial \lambda^{a}}\left(|\sigma|_{\lambda, 1}|g|_{\lambda}|\gamma|_{\lambda}\right)
$$

Lemma 4. If

$$
\sup _{t \in[0, T]} H_{\sigma}(t) \leq M \quad \text { and } \quad \int_{0}^{T} \tilde{H}_{\sigma}(t) d t \leq \tilde{M}
$$

then

$$
\sup _{t \in[0, T]} H_{\rho}(t) \leq M \quad \text { and } \quad \int_{0}^{T} \tilde{H}_{\rho}(t) d t \leq \tilde{M}
$$

for $\rho(t, x):=\int \alpha(v) g(t, x, v) d v$ and $g$ the solution to (5) with initial condition $g_{i}$.

Proof of Lemma 4. By Lemma 3,

$$
\frac{d}{d t}|g|_{\lambda(t), a} \leq a|g|_{\lambda(t), a}+(\lambda-1-K)|g|_{\lambda(t), a+1}+\frac{\partial^{a}}{\partial \lambda^{a}}\left(|\sigma|_{\lambda(t), 1}|g|_{\lambda(t), 1}+|\sigma|_{\lambda(t), 1}|\gamma|_{\lambda(t)}|g|_{\lambda(t)}\right) .
$$


Multiplying each previous inequality by the corresponding $\frac{\alpha(v)}{(a !)^{2}}$ factor, integrating with respect to $v$ and summing with respect to $a$, it holds that

$$
\begin{array}{r}
H_{\rho}^{\prime}(t) \leq \sum_{k \geq 1}|\rho|_{\lambda(t), k} \sum_{a \geq k+1} \frac{C_{a}^{k-1}|\sigma|_{\lambda(t), a-k+2}}{(a !)^{2}}+\left(\lambda-1-K+|\sigma|_{\lambda(t), 1}\right) \sum_{k \geq 1} \frac{|\rho|_{\lambda(t), k}}{((k-1) !)^{2}} \\
+\left(1+|\sigma|_{\lambda(t), 2}\right) \sum_{a \geq 1} \frac{|\rho|_{\lambda, a}}{(a !)^{2}} a^{2}+\sum_{k \geq 0} \frac{|\rho|_{\lambda(t), k}}{k !} \sum_{a \geq 0} \frac{\frac{\partial^{a}}{\partial \lambda^{a}}\left(|\sigma|_{\lambda(t), 1}|\gamma|_{\lambda(t)}\right)}{(a) !(a+k) !} \\
=\sum_{k \geq 1} \frac{|\rho|_{\lambda(t), k}}{(k !)^{2}} \sum_{a \geq 3} \frac{|\sigma|_{\lambda(t), a}}{(a !)^{2}} \frac{k(k !) a(a !)}{(a+k-2) !}+\left(\lambda-1-K+|\sigma|_{\lambda(t), 1}\right) \sum_{k \geq 1} \frac{|\rho|_{\lambda(t), k}}{((k-1) !)^{2}} \\
+\left(1+|\sigma|_{\lambda(t), 2}\right) \sum_{a \geq 1} \frac{a^{2}}{(a !)^{2}}|\rho|_{\lambda, a}+\sum_{k \geq 0} \frac{|\rho|_{\lambda(t), k}}{(k !)^{2}} \sum_{l \geq 0} \frac{|\sigma|_{\lambda(t), l+1}}{((l+1) !)^{2}}(l+1)^{2} \sum_{b \geq 0} \frac{|\gamma|_{\lambda(t), b}}{(b !)^{2}} \frac{k ! l ! b !}{(b+k+l) !} .
\end{array}
$$

Then,

$$
\frac{k ! l ! b !}{(b+k+l) !} \leq \frac{1 \cdots l}{1 \cdots l} \frac{1 \cdots k}{(l+1) \cdots(l+k)} \frac{1 \cdots b}{(l+k+1) \cdots(l+k+b)} \leq 1
$$

and

$$
\frac{k(k !) a(a !)}{(a+k-2) !} \leq \frac{1 \cdots a a}{1 \cdots a(a+1)} 1 \cdot 2 \cdot 3 \cdot 4 \frac{5 \cdots k k}{(a+2) \cdots(a+k-2)} \leq 24, \quad a \geq 3, k \geq 4 .
$$

Similarly for $k=3$,

$$
\frac{k(k !) a(a !)}{(a+k-2) !} \leq 18
$$

And so it only remains $k=1,2$ to consider apart in the right hand side of (8). It gives

$$
\begin{aligned}
H_{\rho}^{\prime}(t) \leq & \left(|\rho|_{\lambda(t), 1}+|\rho|_{\lambda(t), 2}\right) \tilde{H}_{\sigma}(t)+24 H_{\sigma}(t) \sum_{k \geq 3} \frac{|\rho|_{\lambda(t), k}}{(k !)^{2}} \\
& +\left(\lambda_{0}-K+4 H_{\sigma}(t)\right) \tilde{H}_{\rho}(t)+\tilde{\gamma} \tilde{H}_{\sigma}(t) H_{\rho}(t) \\
\leq & (24+\tilde{\gamma}) \tilde{H}_{\sigma}(t) H_{\rho}(t)+\left(\lambda_{0}-K+4 H_{\sigma}(t)\right) \tilde{H}_{\rho}(t) .
\end{aligned}
$$

For $K$ such that

$$
K \geq 2 \lambda_{0}+8 M, \quad K \geq 2(24+\tilde{\gamma}) M\left(\log \frac{M}{h_{0}}\right)^{-1}, \quad T<\frac{\lambda_{0}}{1+K},
$$

and using the second bound of $(7), \sup _{t \in[0, T]} H_{\rho}(t) \leq M$ and $\int_{0}^{T} \tilde{H}_{\rho}(t) d t \leq \tilde{M}$. Such a $K$ exists by assumption (4).

Second step. Let $\sigma$ (resp. $\tilde{\sigma}$ ) in $\tilde{K}, g$ (resp. $\tilde{g}$ ) the solution to (5) with force term $-\partial_{x} \sigma$ $\overline{\left(\operatorname{resp} .-\partial_{x} \tilde{\sigma}\right)}$ and initial datum $g_{i}$, and

$$
(\Phi(\sigma), \Phi(\tilde{\sigma}))=(\rho, \tilde{\rho})=\left(\int \alpha(v) g(\cdot, \cdot, v) d v, \int \alpha(v) \tilde{g}(\cdot, \cdot, v) d v\right) .
$$

Analogously to the proofs of Lemma 2.1 and Lemma $2.2, g-\tilde{g}$ and $\rho-\tilde{\rho}$ satisfy

$$
\begin{array}{r}
\frac{d}{d t}|g-\tilde{g}|_{\lambda(t), a} \leq a|g-\tilde{g}|_{\lambda(t), a}+(\lambda-1-K)|g-\tilde{g}|_{\lambda(t), a+1} \\
\left.+\frac{\partial^{a}}{\partial \lambda^{a}}\left(|\sigma|_{\lambda(t), 1}|g-\tilde{g}|_{\lambda(t), 1}+|\sigma-\tilde{\sigma}|_{\lambda(t), 1}\left(\left|\partial_{v} \tilde{g}\right|_{\lambda(t)}+|\gamma \tilde{g}|_{\lambda(t)}\right)+|\sigma|_{\lambda(t), 1}|\gamma(g-\tilde{g})|_{\lambda(t)}\right)\right),
\end{array}
$$


and

$$
H_{\rho-\tilde{\rho}}^{\prime}(t) \leq \tilde{\gamma} \tilde{M} H_{\rho-\tilde{\rho}}(t)+\left(\lambda_{0}-K+\tilde{\gamma} \tilde{M}+5 M+\tilde{\gamma} M\right) \tilde{H}_{\rho-\tilde{\rho}}(t)+(\tilde{M}+\tilde{\gamma} M) \tilde{H}_{\sigma-\tilde{\sigma}}(t)
$$

For $K>\lambda_{0}+\tilde{\gamma} \tilde{M}+5 M+\tilde{\gamma} M$ and $\bar{K}:=K-\left(\lambda_{0}+\tilde{\gamma} \tilde{M}+5 M+\tilde{\gamma} M\right)$, it holds that

$$
H_{\rho-\tilde{\rho}}(t)+\bar{K} \int_{0}^{t} \tilde{H}_{\rho-\tilde{\rho}}(s) d s \leq(\tilde{M}+\tilde{\gamma} M) e^{\tilde{\gamma} \tilde{M} T} \int_{0}^{t} \tilde{H}_{\sigma-\tilde{\sigma}}(s) d s .
$$

Consequently,

$$
\min \{1, \bar{K}\}\|\rho-\tilde{\rho}\| \leq(\tilde{M}+\tilde{\gamma} M) e^{\tilde{\gamma} \tilde{M} T}\|\sigma-\tilde{\sigma}\|
$$

Under the assumptions (3) and (4) and the choice of $K$ in the interval

$$
] \max \left(2 \lambda_{0}+8 M, \frac{2 M}{\tilde{M}}, \tilde{\gamma} \tilde{M}+5 M+\tilde{\gamma} M+1\right), \frac{\lambda_{0}}{T}-1[,
$$

the map $\Phi$ is a contraction for the $\|\cdot\|$ norm.

Acknowledgement. The authors were partially supported by the I. Newton Institute, Cambridge University.

\section{References}

[1] Bardos, C., Degond, P., Global existence for the Vlasov-Poisson equation in 3-space variables with small initial data. Ann. Inst. H. Poincaré (Analyse non linéaire) 2, nb. 2 (1985) 101-118.

[2] Benachour, S., Analyticité des solutions des équations de Vlasov-Poisson. Ann. Scuol. Norm. Sup. Pisa, Cl. di Sc. 16, nb. 1 (1989) 83-104.

[3] Besse, N., Berthelin, F., Brenier, Y., Bertrand, P., The multi water-bag model for collisionless kinetic equations. Kinetic and Related Models 2, nb. 1 (2009) 39-80.

[4] Bouchut, F., Golse, F., Pallard, C., Classical solutions and the Glassey-Strauss theorem for the 3D Vlasov-Maxwell system. Arch. Ration. Mech. Anal. 170 (2003), no. 1, 1-15.

[5] Di Perna, R., Lions, P.L., Global weak solutions of Vlasov-Maxwell systems. Comm. Pure Appl. Math. 42 (1989) 729-757.

[6] Ghendrih, P., Hauray, M., Nouri, A., Derivation of a gyrokinetic model. Existence and uniqueness of specific stationary solutions. Kinetic and Related Models 2, nb. 4 (2009) 707-725.

[7] R.T. Glassey, The Cauchy problem in kinetic theory, Philadelphia PA, SIAM, 1996.

[8] Glassey, R., Schaeffer, J., The relativistic Vlasov-Maxwell system in two space dimensions, I and II. Arch. Rational Mech. Anal. 141, 331-354 and 355-374 (1998). 
[9] Lions, P.L., Perthame, B., Propagation of moments and regularity for the 3-dimensional Vlasov-Poisson system. Invent. math. 105 (1991) 415-430.

[10] Mouhot, C., Villani, C., On Landau damping. To appear Acta mathematica, Preprint 2009.

[11] K. Pfaffelmoser, Global classical solutions of the Vlasov-Poisson system in three dimensions for general initial data. J. Diff. Eq. 95, 281-303, 1992.

[12] J. Schaeffer, Global existence of smooth solutions to the Vlasov-Poisson system in three dimensions. Comm. P.D.E. 16, 1313-1335, 1991. 